\title{
Atomic Entailment and Atomic Inconsistency and Classical Entailment
}

\author{
T. J. Stepień, L. T. Stepień \\ The Pedagogical University of Cracow, ul. Podchorazych 2, 30 - 084 Krakow, Poland
}

Received: December 01, 2014 / Accepted: December 24, 2014 / Published: February 25, 2015.

\begin{abstract}
In this paper we put forward a new solution of the well-known problem of relevant logics,i.e., we construct an atomic entailment. Hence, we construct a system of predicate calculus based on the atomic entailment. Next, we establish the definition of atomic inconsistency. The atomic inconsistency establishes an infinite class of inconsistent, but non-trivial systems. In this paper we construct the new definition of the classical entailment, into the bargain.
\end{abstract}

Key words: Atomic entailment, atomic inconsistency, classical entailment, relevance

\section{Introduction}

In a number of publications, (see [1] - [7], [9] - [18], [21], [22], [24] - [35], [39] - [45], [53] - [59]), their authors have offered many notions of relevance. Of course, in some publications of these mentioned above, their authors have established the basic properties of the well-known relevant logics. On the other hand, in [14]one can read that although the essence of entailment has been studied from 400 B.C., the problem of establishing such a logic of entailment, which solves the problem of relevance, is still open until now.

Thus, the essential aim is to create such a notion of relevance, which generates a system $\mathrm{S}$ of logic, which satisfies the following condition: this system $\mathrm{S}$ is generated by this notion of relevance, which is defined by a necessary and sufficient condition.

Therefore in this paper we at first construct a new definition of entailment, i.e. the definition of atomic entailment. Then we construct the definition of the system based on the atomic entailment. Next, we build

Corresponding author:L. T. Stepień, The Pedagogical University of Cracow, Kraków, Poland. E-mail: sfstepie@cyf-kr.edu.pl,www.ltstepien.up.krakow.pl

${ }^{2}$ In the next paper we will show that the system $\stackrel{\Pi}{S}$ can be used for the formalization of The Arithmetic System (see [20]). a system $\bar{S}$ of propositional calculus (see [47], [48]) and a system $\stackrel{\Pi}{S}$ of predicate calculus, which are based on the atomic entailment (see [49], [51], [52])2. Besides, in this paper, we give also the new definition of the classical entailment.

\section{Notational Preliminaries}

Let $\rightarrow, \sim, \vee, \wedge, \equiv$ denote the connectives of implication, negation, disjunction, conjunction and equivalence, respectively. We use $\Rightarrow, \neg, \Leftrightarrow, \&, \mathbb{V}, \forall, \exists$ as metalogical symbols. Next $A t_{0}=\left\{p, p_{1}, p_{2}, \ldots, q, q_{1}, q_{2}, \ldots, s, s_{1}, s_{2}, \ldots t, \ldots\right\}$

denotes the set of all propositional variables. $S_{0}$ is the set of all well-formed formulas, which are built in the usual manner from propositional variables and by means of logical connectives. $P_{0}(\phi)$ denotes the set of all propositional variables occuring in $\phi\left(\phi \in S_{0}\right)$. $R_{S_{0}}$ denotes the set of all rules over $S_{0}$. Hence, for every $r \in R_{S_{0}},\langle\Pi, \phi\rangle \in r$, where $\Pi \subseteq S_{0} \quad$ and $\phi \in S_{0}$ and $\Pi$ is a set of premisses and $\phi$ is a conclusion. Hence, $r_{*}^{0}$ denotes here the rule of simultaneous substitution for propositional variables. $\langle\{\phi\}, \psi\rangle \in r_{*}^{0} \Leftrightarrow\left[h^{e}(\phi)=\psi\right]$, where $h^{e}$ is the extension of the mapping $e: A t_{0} \rightarrow S_{0}\left(e \in \varepsilon_{*}^{0}\right)$ 
to endomorphism $h^{e}: S_{0} \rightarrow S_{0}$, where

$$
\begin{gathered}
h^{e}(\phi)=e(\phi), \text { for } \phi \in A t_{0} \\
h^{e}(\sim \phi)=\sim h^{e}(\phi) \\
h^{e}(\phi F \psi)=h^{e}(\phi) F h^{e}(\psi),
\end{gathered}
$$

for $F \in\{\rightarrow, \vee, \wedge, \equiv\}$ and for every $\phi, \psi \in S_{0}$.

Thus, $\varepsilon_{*}^{0}$ is a class of functions $e: A t_{0} \rightarrow S_{0}$ (for details, see [36]) (cf. [19]). $r_{0}^{0}$ denotes here the Modus Ponens rule in propositional calculus. $R_{0 *}=\left\{r_{0}^{0}, r_{*}^{0}\right\}$ (for details, see [19], [36]). A logical matrix is a pair $\mathfrak{M}=\left\{U, U^{\prime}\right\}, U$ is an abstract algebra and $U^{\prime}$ is a subset of the universe $U$, i.e. $U^{\prime} \subseteq U$. Any $a \in U^{\prime}$ is called a distinguished element of the matrix $\mathfrak{M} . E(\mathfrak{M})$ is the set of all formulas valid in the matrix $\mathfrak{M} . \mathfrak{M}_{2}$ denotes the classical two-valued matrix. Hence, $Z_{2}$ is the set of all formulas valid in the classical matrix $\mathfrak{M}_{2}$ (see [19], [36]).

The symbols $x_{1}, x_{2}, \ldots$ are individual variables. $a_{1}, a_{2}, \ldots$ are individual constants. $V$ is the set of all individual variables. $P_{i}^{n}(i, n \in \mathcal{N}=\{1,2, \ldots\})$ are $n$-ary predicate letters. The symbols $f_{i}^{n}(i, n \in \mathcal{N})$ are $\mathrm{n}$-ary function letters. The symbols $\wedge x_{k}, \vee x_{k}$ are quantifiers. $\Lambda x_{k}$ is the universal quantifier and $\vee x_{k}$ is the existential quantifier. The function letters, applied to the individual variables and individual constants, generate terms. The symbols $t_{1}, t_{2}, \ldots$ areterms. $T$ is the set of all terms. The predicate letters, applied to terms, yield simple formulas, i.e. if $P_{i}^{k}$ is a predicate letter and $t_{1}, \ldots, t_{k}$ are terms, then $P_{i}^{k}\left(t_{1}, \ldots, t_{n}\right)$ is a simple formula. Smp is the set of all simple formulas. Next, $A t_{1}$ is the set of all atomic formulas, where $A t_{1}=\left\{P_{i}^{k}\left(x_{j_{1}}, \ldots, x_{j_{k}}\right)\right.$ :

$\left.k, i, j_{1}, \ldots, j_{k} \in \mathcal{N}\right\}$. At last $S_{1}$ is the set of all well-formed formulas. $F V(\phi)$ denotes the set of all free variables occuring in $\phi$, where $\phi \in S_{1}$. $x_{k} \in F f\left(t_{m}, \phi\right)$ expresses that $x_{k}$ is free for term $t_{m}$ in $\phi$. By $x_{k} / t_{m}$ we denote the substitution of the term $t_{m}$ for the individual variable $x_{k} . P_{1}(\phi)$ denotes the set of all predicate letters occuring in $\phi\left(\phi \in S_{1}\right)$. If $F V(\phi)=\left\{x_{1}, \ldots, x_{k}\right\}$, then $\wedge \phi=$ $\wedge x_{1} \ldots \wedge x_{k} \phi$.

$R_{S_{1}}$ denotes the set of all rules over $S_{1}$. Hence, for every $r \in R_{S_{1}}, \quad\langle\Pi, \phi\rangle \in r$, where $\Pi \subseteq S_{1}$ and $\phi \in S_{1}$ and $\Pi$ is a set of premisses and $\phi$ is a conclusion. Hence, $r_{*}^{1}$ denotes here the rule of simultaneous substitution for predicate letters. $\langle\{\phi\}, \psi\rangle \in r_{*}^{1} \Leftrightarrow\left[h^{e}(\phi)=\psi\right]$, where $h^{e}$ is the extension of the mapping $e: S m p \rightarrow S_{1}\left(e \in \varepsilon_{*}^{1}\right)$ to endomorphism $h^{e}: S_{1} \rightarrow S_{1}$, where

$$
\begin{gathered}
h^{e}(\phi)=e(\phi), \text { for } \phi \in S m p \\
h^{e}(\sim \phi)=\sim h^{e}(\phi) \\
h^{e}(\phi F \psi)=h^{e}(\phi) F h^{e}(\psi), \\
\text { for } F \in\{\rightarrow, \vee, \wedge, \equiv\} \\
h^{e}\left(\wedge x_{k} \phi\right)=\wedge x_{k} h^{e}(\phi) \\
h^{e}\left(\vee x_{k} \phi\right)=\vee x_{k} h^{e}(\phi),
\end{gathered}
$$

for every $\phi, \psi \in S_{1}$ and $k \in \mathcal{N}$ (for details, see [37], [38]).

Next, $r_{0}^{1}$ denotes the Modus Ponens rule in predicate calculus, $r_{+}^{1}$ denotes the generalization rule. $R_{0+}=\left\{r_{0}^{1}, r_{+}^{1}\right\}, R_{0 *+}=\left\{r_{0}^{1}, r_{*}^{1}, r_{+}^{1}\right\}$. We write $X \subset Y$, if $X \subseteq Y$ and $X \neq Y$.

We assume here that for every $\alpha \in S_{1}$, if $F V(\alpha)=\left\{x_{1}, \ldots, x_{n}\right\}$, then $\alpha^{*}=\bigvee x_{1} \ldots \bigvee x_{n} \sim \alpha$. Hence, for every $\alpha \in S_{1}$, if $F V(\alpha)=\emptyset$, then $\alpha^{*}=\sim \alpha$. Analogically, for every $\alpha \in S_{0}, \alpha^{*}=\sim \alpha$.

Finally, for any $X \subseteq S_{i}$ and $R \subseteq R_{S_{i}}, C n_{i}(R, X)$ is the smallest subset of $S_{i}$ containing $X$ and closed under the rules $R \subseteq R_{S_{i}}$, where $i \in\{0,1\}$. The couple $\langle R, X\rangle$ is called a system, whenever $R \subseteq R_{S_{i}}$ and $X \subseteq S_{i}$ and $i \in\{0,1\}$. Syst $\cap A_{0}$ denotes here the class of all systems $\langle R, X\rangle$, which are based on an atomic entailment, where $R \subseteq R_{S_{0}}$ and $X \subseteq$ $S_{0}$. Syst $\cap A_{1}$ denotes here the class of all systems $\langle R, X\rangle$, which are based on an atomic entailment, where $R \subseteq R_{S_{1}}$ and $X \subseteq S_{1}$. Syst $\cap C_{1}$ denotes here the class of all systems $\langle R, X\rangle$, which are based on a classical entailment, where $R \subseteq R_{S_{1}}$ and $X \subseteq S_{1}$. $\phi \mid \frac{A_{0}}{R, X} \psi$ denotes that $\psi$ results atomically from $\phi$ on the ground of the system $\langle R, X\rangle$, where $R \subseteq R_{S_{0}}$ and $X \subseteq S_{0}$. Next, $\phi \mid \frac{A_{1}}{R, X} \psi$ denotes that $\psi$ results atomically from $\phi$ on the ground of the system 
$\langle R, X\rangle$, where $R \subseteq R_{S_{1}}$ and $X \subseteq S_{1}$. At last, $\phi \mid \frac{C_{1}}{R, X} \psi$ denotes that $\psi$ results classically from $\phi$ on the ground of the system $\langle R, X\rangle$, where $R \subseteq R_{S_{1}}$ and $X \subseteq S_{1} . \bar{S}_{1}=\left\{\phi \in S_{1}: F V(\phi)=\emptyset\right\}, S_{1}^{*}$ denotes the set of all formulas, which are in normal form (see [19] pp. 35 - 42 and 130 - 132, [20] pp. 214 - 222 and [37] pp. 146 - 149).

Definition 2.1. The function $j: S_{1} \rightarrow S_{0}$, is defined, as follows:

$$
\begin{gathered}
j\left(P_{k}^{n}\left(t_{1}, \ldots, t_{n}\right)\right)=p_{k}\left(p_{k} \in A t_{0}\right) \\
j(\sim \phi)=\sim j(\phi) \\
j(\phi F \psi)=j(\phi) F j(\psi), \text { for } F \in\{\rightarrow, \vee, \wedge, \equiv\} \\
j\left(\wedge x_{k} \phi\right)=j\left(\vee x_{n} \phi\right)=j(\phi) .
\end{gathered}
$$

By $\langle R, X\rangle \in C n s^{A}$ we denote here the well-known notion of the absolute consistency (see [36] and [37]). Thus,

Definition 2.2. $\langle R, X\rangle \in C n s^{A} \Leftrightarrow \operatorname{Cn}(R, X) \neq S_{i}$, where $R \subseteq R_{S_{i}}, X \subseteq S_{i}$ and $i \in\{0,1\}$.

\section{Classical Entailment}

Definition 3.1. Let $C n_{1}(R, X)=L \neq \emptyset \quad$ and $\phi, \psi \in S_{1}$. Then $\phi \mid \frac{C_{1}}{R, X} \psi$ iff the following conditions are satisfied

(1) $\left(\forall e \in \varepsilon_{*}^{1}\right)\left[h^{e}(\wedge \phi) \in L \Rightarrow h^{e}(\psi) \in L\right]$

(2) $\left(\forall e \in \varepsilon_{*}^{1}\right)\left[h^{e}\left(\left(\psi^{*} \rightarrow \phi^{*}\right) \rightarrow \phi^{*}\right) \in L \Rightarrow\right.$ $\left.h^{e}\left(\phi^{*}\right) \in L\right]$.

Definition 3.2. $\langle R, X\rangle \in$ Syst $\cap C_{1}$ iff the following condition is satisfied:

$\left(\forall \phi, \psi \in S_{1}\right)\left[\wedge \phi \rightarrow \psi \in C n_{1}(R, X) \Leftrightarrow \phi \mid \frac{C_{1}}{R, X} \psi\right]$.

\section{The classical predicate logic}

Let $A_{2}$ denote the set of axioms of the classical predicate logic. Hence, $\left\langle R_{0+}, A_{2}\right\rangle$ denotes the classical predicate calculus, where $\operatorname{Cn}\left(R_{0+}, A_{2}\right)=L_{2}$ (see [20] and [37]).

Thus, (see [37] p.57, p.71):

Theorem 4.1. $C n_{1}\left(R_{0 *+}, L_{2}\right)=L_{2}$.

Now we notice that on the ground of the classical predicate calculus, the following theorem is valid (cf. [20] pp. 222 - 223):

Theorem 4.2. (on the extensionality of logical expressions). Let $x_{1}, \ldots, x_{n}, y_{1}, \ldots, y_{l}$ be all the free variables, which occur in the expressions $\alpha$ and $\beta$, and let $C^{\alpha}$ be any expression that contains $\alpha$ or an expression obtained from $\alpha$ by the substitution for the variables $x_{1}, \ldots, x_{n}$ of some other variables different from the bound variables occurring in the expressions $\alpha$ or $\beta$, and let $C^{\beta}$ differ from $C^{\alpha}$ only in that in certain places (unnecessarily in all these places) in which in $C^{\alpha}$ there occurs $\alpha$ or an expression obtained from $\alpha$ by a substitution for the variables $x_{1}, \ldots, x_{n}$, in the corresponding places in $C^{\beta}$ there occurs $\beta$ or an expression obtained from $\beta$ by an appropriate substitution, while the variables $y_{1}, \ldots, y_{l}$ are all the free variables in $C^{\alpha}$ and $C^{\beta}$. Then the sentence:

$$
\wedge \ldots y_{1}, \ldots, y_{l}\left(\wedge x_{1}, \ldots, x_{n}(\alpha \equiv \beta) \rightarrow\left(C^{\alpha} \equiv C^{\beta}\right)\right)
$$

is a theorem in $L_{2}$.

\section{Atomic Entailment}

In [57] one can read that Lewis told that from his very first contact with the logic of "Principia Mathematica", he had been bothered by the paradoxes of material implication. As Whitehead and Russell have it written, a true proposition is implied by arbitrary (true or false) proposition, while a false proposition implies arbitrary (true or false) proposition. Aiming at avoiding these consequences of the material conditional, Lewis wrote his first paper devoted to logic (in this current paper, the Lewis' paper is as [25]). At first, it ought to be noticed here that the results contained in [1] - [7], [9] - [18], [21], [22], [24] - [35], [39] - [45], [53] - [59], and in the other papers, have essentially contributed to the better understanding of the problem of relevance. Thus (see [47], [48], [49], [51], [52]):

Definition 5.1. Let $C n_{0}(R, X)=L \neq \varnothing \quad$ and $\phi, \psi \in S_{0}$. Then $\phi \mid \frac{A_{0}}{R, X} \psi$ iff the following conditions 
are satisfied:

(1) $\left(\forall e \in \varepsilon_{*}^{0}\right)\left[h^{e}(\phi) \in L \Rightarrow h^{e}(\psi) \in L\right.$

$$
\left.\& P_{0}\left(h^{e}(\phi)\right) \subseteq P_{0}\left(h^{e}(\psi)\right)\right]
$$

(2) $\left(\forall e \in \varepsilon_{*}^{0}\right)\left[h^{e}\left(\left(\psi^{*} \rightarrow \phi^{*}\right) \rightarrow \phi^{*}\right) \in L \Rightarrow\right.$ $\left.h^{e}\left(\phi^{*}\right) \in L \& P_{0}\left(h^{e}\left(\psi^{*}\right)\right) \subseteq P_{0}\left(h^{e}\left(\phi^{*}\right)\right)\right]$.

Definition 5.2. $\langle R, X\rangle \in$ Syst $\cap A_{0}$ iff the following condition is satisfied:

$\left(\forall \psi, \phi \in S_{0}\right)\left[\phi \rightarrow \psi \in C n_{0}(R, X) \Leftrightarrow \phi \mid \frac{A_{0}}{R, X} \psi\right]$.

Definition 5.3. Let $C n_{1}(R, X)=L \neq \varnothing \quad$ and $\phi, \psi \in S_{1}$. Then $\phi \mid \frac{A_{1}}{R, X} \psi$ iff the following conditions are satisfied:

(1) $\left(\forall e \in \varepsilon_{*}^{1}\right)\left[h^{e}(\Lambda \phi) \in L \Rightarrow h^{e}(\psi) \in L\right.$

$\left.\& P_{1}\left(h^{e}(\Lambda \phi)\right) \subseteq P_{1}\left(h^{e}(\psi)\right)\right]$

(2) $\left(\forall e \in \varepsilon_{*}^{1}\right)\left[h^{e}\left(\left(\psi^{*} \rightarrow \phi^{*}\right) \rightarrow \phi^{*}\right) \in L \Rightarrow\right.$ $\left.h^{e}\left(\phi^{*}\right) \in L \& P_{1}\left(h^{e}\left(\psi^{*}\right)\right) \subseteq P_{1}\left(h^{e}\left(\phi^{*}\right)\right)\right]$.

Definition 5.4. $\langle R, X\rangle \in$ Syst $\cap A_{1}$ iff the following condition is satisfied:

$\left(\forall \psi, \phi \in S_{1}\right)\left[\wedge \phi \rightarrow \psi \in C n_{1}(R, X) \Leftrightarrow \phi \mid \frac{A_{1}}{R, X} \psi\right]$.

\section{Atomic Inconsistency}

By Syst $\cap A I N C$ we denote here the class of all systems $\langle R, X\rangle$, which have the property of atomic inconsistency (see also [8], [23], [60]), where $R \subseteq R_{S_{i}}$ and $X \subseteq S_{i}$ and $i \in\{0,1\}$.

Definition 6.1. Let $i \in\{0,1\}$ and $\alpha \in S_{i}$. Then $S_{i \alpha}=\left\{\phi \in S_{i}: P_{i}(\phi) \subseteq P_{i}(\alpha)\right\}$.

Definition 6.2. Let $R \subseteq R_{S_{i}}$ and $X \subseteq S_{i}$ and $i \in\{0,1\}$. Then

$$
\langle R, X\rangle \in \text { Syst } \cap \text { AINC } \Leftrightarrow
$$

$\left(\forall \alpha \in S_{i}\right)\left\{\left[S_{i \alpha} \subseteq \operatorname{Cn}(R, X \cup\{\alpha, \sim \alpha\})\right] \&\right.$

$\left(\forall \beta \in S_{i}\right)\left[P_{i}(\beta) \nsubseteq P_{i}(\alpha) \Rightarrow\right.$

$\beta \notin \operatorname{Cn}(R, X \cup\{\alpha, \sim \alpha\}) \mathbb{V}$

$\sim \beta \notin \operatorname{Cn}(R, X \cup\{\alpha, \sim \alpha\})]\}$.

\section{System $\bar{S}$}

Let us take the matrix

$\mathfrak{M}_{D}=\left\langle\{0,1,2\},\{1,2\}, f_{D}^{\rightarrow}, f_{\bar{D}}^{\equiv}, f_{D}^{\vee}, f_{D}^{\wedge}, f_{D}^{\sim}\right\rangle$, where:

\begin{tabular}{l|l|l|l|}
$f_{D} \vec{n}$ & 0 & 1 & 2 \\
\hline 0 & 1 & 1 & 1 \\
\hline 1 & 0 & 1 & 0 \\
\hline 2 & 0 & 1 & 2 \\
\hline
\end{tabular}

\begin{tabular}{|l|l|l|l}
$f_{D} \equiv$ & 0 & 1 & 2 \\
\hline 0 & 1 & 0 & 0 \\
\hline 1 & 0 & 1 & 0 \\
\hline 2 & 0 & 0 & 2 \\
\hline
\end{tabular}

\begin{tabular}{l|l|l|l|}
\hline$f_{D}^{\vee}$ & 0 & 1 & 2 \\
\hline 0 & 0 & 1 & 0 \\
\hline 1 & 1 & 1 & 1 \\
\hline 2 & 0 & 1 & 2
\end{tabular}

\begin{tabular}{|l|l|l|l}
\hline$f_{D}^{\wedge}$ & 0 & 1 & 2 \\
\hline 0 & 0 & 0 & 0 \\
\hline 1 & 0 & 1 & 1 \\
\hline 2 & 0 & 1 & 2
\end{tabular}

In [47] (see [48]) we have defined the system $\bar{S}$ as follows:

Definition 7.1. $\bar{S}=\left\langle R_{0 *}, T_{D}\right\rangle$, where $T_{D}=E\left(\mathfrak{M}_{D}\right)$.

Thus, the system $\bar{S}$ is the logic that is obtained from the set of valid formulas in the matrix $\mathfrak{M}_{D}$, by the rules of substitution and detachment.

It should be noticed here that the matrix $\mathfrak{M}_{D}^{\prime}=$ $\left\langle\{0,1,2\},\{1,2\}, f_{D}, f_{D}^{\sim}\right\rangle$ was investigated by $\mathrm{B}$. Sobocinski (see [46], [47]).

Next, in [47] we have proved the following:

Theorem 7.2. Let $\phi, \psi \in S_{0}$ and

$$
\left(\exists e \in \varepsilon_{*}^{0}\right)\left[h^{e}(\phi) \in T_{D}\right] \text {. }
$$

Then $\phi \rightarrow \psi \in C n_{0}\left(R_{0 *}, T_{D}\right)$ iff

$$
\left(\forall e \in \varepsilon_{*}^{0}\right)\left[h^{e}(\phi) \in T_{D} \Rightarrow h^{e}(\psi) \in T_{D}\right.
$$$$
\left.\& P_{0}\left(h^{e}(\phi)\right) \subseteq P_{0}\left(h^{e}(\psi)\right)\right] \text {. }
$$

Theorem 7.3. The system $\bar{S}$ is axiomatizable.

\section{System $\stackrel{\sqcap}{S}$}

At first we define the set $L_{D}$, putting:

Definition 8.1. $L_{D}=\left\{\phi \in S_{1}: j(\phi) \in T_{D} \& \phi \in L_{2}\right\}$.

Next, we define the system $\stackrel{\Pi}{S}$, as follows:

Definition 8.2. $\stackrel{\Pi}{S}=\left\langle R_{0+}, L_{D}\right\rangle$.

By Theorem 4.1 and by Definition 8.1, one obtains:

Corollary 8.3. $\mathrm{Cn}_{1}\left(R_{0 *+}, L_{D}\right)=L_{D}$.

By Definition 8.1 and by Corollary 8.3, we get 
Corollary 8.4. Let $\alpha, \beta, \gamma, \phi, \psi, \delta \in S_{1}$ and $Q_{i} \in\left\{\wedge x_{i}, \bigvee x_{i}\right\}$ and $i, k, s \in \mathcal{N}$. Then the following formulas belong to $L_{D}$ :

(1) $\alpha \rightarrow \alpha$

(2) $\alpha \rightarrow[(\alpha \rightarrow \beta) \rightarrow \beta]$

(3) $(\alpha \rightarrow \beta) \rightarrow[(\beta \rightarrow \gamma) \rightarrow(\alpha \rightarrow \gamma)]$

(4) $[\alpha \rightarrow(\beta \rightarrow \gamma)] \rightarrow[\beta \rightarrow(\alpha \rightarrow \gamma)]$

(5) $[\alpha \rightarrow(\alpha \rightarrow \beta)] \rightarrow(\alpha \rightarrow \beta)$

(6) $\{[(\beta \rightarrow \gamma) \rightarrow(\alpha \rightarrow \gamma)] \rightarrow \delta\} \rightarrow[(\alpha \rightarrow \beta) \rightarrow \delta]$

(7) $[\alpha \rightarrow(\beta \rightarrow \gamma)] \rightarrow\{(\delta \rightarrow \beta) \rightarrow[\alpha \rightarrow(\delta \rightarrow \gamma)]\}$

(8) $[\alpha \rightarrow(\beta \rightarrow \gamma)] \rightarrow[(\alpha \rightarrow \beta) \rightarrow(\alpha \rightarrow \gamma)]$

(9) $(\beta \rightarrow \gamma) \rightarrow[(\alpha \rightarrow \beta) \rightarrow(\alpha \rightarrow \gamma)]$

(10) $(\beta \rightarrow \gamma) \rightarrow\{(\alpha \rightarrow \beta) \rightarrow[(\gamma \rightarrow \delta) \rightarrow$ $(\alpha \rightarrow \delta)]\}$

(11) $\sim \sim \alpha \rightarrow \alpha$

(12) $\alpha \rightarrow \sim \sim \alpha$

(13) $(\sim \alpha \rightarrow \alpha) \rightarrow \alpha$

(14) $(\alpha \rightarrow \sim \alpha) \rightarrow \sim \alpha$

(15) $(\sim \sim \alpha \rightarrow \sim \sim \beta) \rightarrow(\alpha \rightarrow \beta)$

(16) $(\alpha \rightarrow \sim \beta) \rightarrow(\sim \sim \alpha \rightarrow \sim \beta)$

(17) $(\alpha \rightarrow \beta) \rightarrow(\alpha \rightarrow \sim \sim \beta)$

(18) $(\alpha \rightarrow \sim \beta) \rightarrow(\beta \rightarrow \sim \alpha)$

(19) $(\sim \beta \rightarrow \sim \sim \alpha) \rightarrow(\sim \beta \rightarrow \alpha)$

(20) $(\sim \alpha \rightarrow \beta) \rightarrow(\sim \beta \rightarrow \alpha)$

(21) $\alpha \rightarrow \sim(\alpha \rightarrow \sim \alpha)$

(22) $(\sim \alpha \rightarrow \sim \sim \alpha) \rightarrow \alpha$

(23) $(\alpha \rightarrow \beta) \rightarrow(\sim \beta \rightarrow \sim \alpha)$

(24) $(\alpha \rightarrow \beta) \rightarrow[(\alpha \rightarrow \sim \beta) \rightarrow \sim \alpha]$

(25) $(\sim \alpha \rightarrow \beta) \rightarrow[(\sim \beta \rightarrow \sim \alpha) \rightarrow \sim \sim \beta]$

(26) $(\sim \alpha \rightarrow \beta) \rightarrow[(\alpha \rightarrow \beta) \rightarrow \beta]$

(27) $\alpha \rightarrow[\beta \rightarrow \sim(\alpha \rightarrow \sim \beta)]$

(28) $\alpha \equiv \alpha$

(29) $\alpha \equiv \sim \sim \alpha$

(30) $\sim \sim \alpha \equiv \alpha$

(31) $(\alpha \rightarrow \beta) \rightarrow[(\beta \equiv \gamma) \rightarrow(\alpha \rightarrow \gamma)]$

(32) $(\alpha \equiv \beta) \rightarrow[(\beta \equiv \gamma) \rightarrow(\alpha \rightarrow \gamma)]$

(33) $(\beta \rightarrow \alpha) \rightarrow[(\beta \equiv \gamma) \rightarrow(\gamma \rightarrow \alpha)]$

(34) $(\alpha \equiv \beta) \rightarrow(\alpha \rightarrow \beta)$

(35) $(\alpha \equiv \beta) \rightarrow(\beta \rightarrow \alpha)$

(36) $(\alpha \rightarrow \beta) \rightarrow[(\beta \rightarrow \alpha) \rightarrow(\alpha \equiv \beta)]$

(37) $(\alpha \equiv \beta) \rightarrow[(\alpha \rightarrow \gamma) \equiv(\beta \rightarrow \gamma)]$
(38) $(\alpha \equiv \beta) \rightarrow[(\gamma \equiv \alpha) \equiv(\gamma \equiv \beta)]$

(39) $(\alpha \equiv \beta) \rightarrow(\beta \equiv \alpha)$

(40) $\alpha \rightarrow(\alpha \vee \beta)$, if $P_{1}(\alpha)=P_{1}(\alpha \vee \beta)$

(41) $\alpha \rightarrow(\beta \vee \alpha)$, if $P_{1}(\alpha)=P_{1}(\beta \vee \alpha)$

(42) $(\alpha \vee \beta) \rightarrow[(\alpha \rightarrow \beta) \rightarrow \beta]$

(43) $(\alpha \rightarrow \beta) \rightarrow[(\alpha \vee \gamma) \rightarrow(\gamma \vee \beta)]$

(44) $(\alpha \rightarrow \beta) \rightarrow[(\alpha \vee \gamma) \rightarrow(\beta \vee \gamma)]$

(45) $(\alpha \rightarrow \beta) \rightarrow[(\gamma \vee \alpha) \rightarrow(\gamma \vee \beta)]$

(46) $[\alpha \vee(\beta \vee \gamma)] \rightarrow[(\alpha \vee \beta) \vee \gamma]$

(47) $[\alpha \vee(\beta \vee \gamma)] \rightarrow[\alpha \vee(\gamma \vee \beta)]$

(48) $[\alpha \vee(\gamma \vee \beta)] \rightarrow[(\alpha \vee \beta) \vee \gamma]$

(49) $[\alpha \vee(\beta \vee \gamma)] \rightarrow[\beta \vee(\alpha \vee \gamma)]$

(50) $[\gamma \vee(\alpha \vee \beta)] \rightarrow[\beta \vee(\gamma \vee \alpha)]$

(51) $[\beta \vee(\gamma \vee \alpha)] \rightarrow[\beta \vee(\alpha \vee \gamma)]$

(52) $[(\beta \vee \alpha) \vee \gamma] \rightarrow[\beta \vee(\alpha \vee \gamma)]$

(53) $(\alpha \rightarrow \beta) \vee(\beta \rightarrow \alpha)$

(54) $(\alpha \rightarrow \beta) \rightarrow\{(\gamma \rightarrow \beta) \rightarrow[(\alpha \vee \gamma) \rightarrow \beta]\}$

(55) $\sim \alpha \vee \alpha$

(56) $\alpha \vee \sim \alpha$

(57) $(\alpha \vee \beta) \rightarrow(\sim \beta \rightarrow \alpha)$

(58) $(\alpha \vee \beta) \rightarrow(\sim \alpha \rightarrow \beta)$

(59) $(\sim \alpha \vee \beta) \rightarrow(\alpha \rightarrow \beta)$

(60) $\alpha \rightarrow(\alpha \wedge \alpha)$

(61) $(\alpha \wedge \beta) \rightarrow(\beta \wedge \alpha)$

(62) $\alpha \rightarrow[\beta \rightarrow(\alpha \wedge \beta)]$

(63) $[(\alpha \wedge \beta) \rightarrow(\beta \rightarrow \gamma)] \rightarrow[(\alpha \wedge \beta) \rightarrow \gamma]$

(64) $[\alpha \rightarrow(\beta \rightarrow \gamma)] \rightarrow[(\alpha \wedge \beta) \rightarrow \gamma]$

(65) $[(\alpha \wedge \beta) \rightarrow \gamma] \rightarrow[\alpha \rightarrow(\beta \rightarrow \gamma)]$

(66) $[(\alpha \rightarrow \beta) \wedge \alpha] \rightarrow \beta$

(67) $[(\alpha \wedge \gamma) \rightarrow \beta] \rightarrow[(\alpha \wedge \gamma) \rightarrow(\beta \wedge \gamma)]$

(68) $(\alpha \rightarrow \beta) \rightarrow[(\gamma \wedge \alpha) \rightarrow(\gamma \wedge \beta)]$

(69) $(\alpha \rightarrow \beta) \rightarrow\{(\alpha \rightarrow \gamma) \rightarrow[\alpha \rightarrow(\beta \wedge \gamma)]\}$

(70) $[(\alpha \rightarrow \gamma) \wedge(\beta \rightarrow \delta)] \rightarrow[(\alpha \wedge \beta) \rightarrow(\gamma \wedge \delta)]$

(71) $[(\alpha \rightarrow \gamma) \wedge(\beta \rightarrow \delta)] \rightarrow[(\beta \wedge \alpha) \rightarrow(\delta \wedge \gamma)]$

(72) $[(\alpha \rightarrow \beta) \wedge(\alpha \rightarrow \gamma)] \rightarrow[\alpha \rightarrow(\beta \wedge \gamma)]$

(73) $[(\alpha \wedge \beta) \wedge \gamma] \rightarrow\{[(\alpha \wedge \beta) \wedge \gamma] \wedge \beta\}$

(74) $\{[(\alpha \wedge \beta) \wedge \gamma] \wedge \beta\} \rightarrow[(\alpha \wedge \gamma) \wedge \beta]$

(75) $\sim(\alpha \wedge \sim \alpha)$

(76) $\sim(\sim \alpha \wedge \alpha)$

(77) $\sim(\alpha \rightarrow \beta) \rightarrow(\alpha \wedge \sim \beta)$

(78) $[\sim(\alpha \wedge \sim \beta) \wedge \alpha] \rightarrow \beta$ 
(79) $[\alpha \wedge \sim(\alpha \wedge \sim \beta)] \rightarrow \beta$

(80) $(\alpha \wedge \beta) \rightarrow(\sim \sim \alpha \wedge \sim \sim \beta)$

(81) $(\alpha \wedge \beta) \rightarrow \sim(\alpha \rightarrow \sim \beta)$

(82) $(\alpha \wedge \sim \sim \beta) \rightarrow(\alpha \wedge \beta)$

(83) $\sim(\alpha \rightarrow \sim \beta) \rightarrow(\alpha \wedge \beta)$

(84) $(\alpha \rightarrow \sim \sim \beta) \rightarrow \sim(\alpha \wedge \sim \beta)$

(85) $(\alpha \rightarrow \sim \beta) \rightarrow \sim(\alpha \wedge \beta)$

(86) $(\alpha \rightarrow \beta) \equiv(\sim \beta \rightarrow \sim \alpha)$

(87) $(\alpha \equiv \beta) \equiv(\sim \alpha \equiv \sim \beta)$

(88) $(\alpha \wedge \beta) \equiv(\beta \wedge \alpha)$

(89) $[\alpha \wedge(\beta \wedge \gamma)] \equiv[(\alpha \wedge \beta) \wedge \gamma]$

(90) $[(\alpha \equiv \beta) \wedge(\gamma \equiv \delta)] \rightarrow[(\alpha \rightarrow \gamma) \equiv(\beta \rightarrow \delta)]$

(91) $(\alpha \equiv \beta) \rightarrow[(\beta \rightarrow \alpha) \wedge(\alpha \rightarrow \beta)]$

(92) $(\alpha \equiv \beta) \rightarrow[(\alpha \rightarrow \beta) \wedge(\beta \rightarrow \alpha)]$

(93) $(\alpha \wedge \alpha) \equiv \alpha$

(94) $(\alpha \equiv \beta) \rightarrow[(\alpha \wedge \gamma) \equiv(\beta \wedge \gamma)]$

(95) $(\alpha \equiv \beta) \rightarrow[(\gamma \wedge \alpha) \equiv(\gamma \wedge \beta)]$

(96) $[(\alpha \equiv \beta) \wedge(\gamma \equiv \delta)] \rightarrow[(\alpha \equiv \gamma) \equiv(\beta \equiv \delta)]$

(97) $[(\alpha \rightarrow \gamma) \wedge(\gamma \rightarrow \alpha)] \rightarrow(\alpha \equiv \gamma)$

(98) $[(\alpha \equiv \beta) \wedge(\gamma \equiv \delta)] \rightarrow[(\alpha \wedge \gamma) \equiv(\beta \wedge \delta)]$

(99) $[(\alpha \equiv \beta) \wedge(\beta \equiv \gamma)] \rightarrow[(\alpha \rightarrow \gamma) \wedge(\gamma \rightarrow \alpha)]$

(100) $(\alpha \vee \alpha) \equiv \alpha$

(101) $(\alpha \vee \beta) \equiv(\beta \vee \alpha)$

(102) $(\alpha \equiv \beta) \rightarrow[(\gamma \vee \alpha) \equiv(\gamma \vee \beta)]$

(103) $(\alpha \vee \beta) \equiv(\sim \alpha \rightarrow \beta)$

(104) $(\alpha \rightarrow \beta) \equiv(\sim \alpha \vee \beta)$

$(105)[(\alpha \equiv \beta) \wedge(\gamma \equiv \delta)] \rightarrow[(\alpha \vee \gamma) \equiv(\beta \vee \delta)]$

(106) $\sim(\alpha \wedge \beta) \equiv(\alpha \rightarrow \sim \beta)$

(107) $\sim(\alpha \wedge \beta) \equiv(\beta \rightarrow \sim \alpha)$

(108) $(\alpha \vee \beta) \rightarrow \sim(\sim \alpha \wedge \sim \beta)$

(109) $(\sim \alpha \wedge \sim \beta) \rightarrow \sim(\alpha \vee \beta)$

(110) $\sim(\sim \alpha \vee \sim \beta) \rightarrow(\alpha \wedge \beta)$

(111) $\sim(\alpha \wedge \beta) \rightarrow(\sim \alpha \vee \sim \beta)$

(112) $\sim(\alpha \vee \beta) \rightarrow(\sim \alpha \wedge \sim \beta)$

(113) $\sim(\sim \alpha \wedge \sim \beta) \rightarrow(\alpha \vee \beta)$

(114) $(\alpha \wedge \beta) \rightarrow \sim(\sim \alpha \vee \sim \beta)$

(115) $(\sim \alpha \vee \sim \beta) \rightarrow \sim(\alpha \wedge \beta)$

(116) $(\alpha \vee \beta) \equiv \sim(\sim \alpha \wedge \sim \beta)$

(117) $(\alpha \wedge \beta) \equiv \sim(\sim \alpha \vee \sim \beta)$

(118) $(\alpha \wedge \beta) \equiv \sim(\alpha \rightarrow \sim \beta)$

(119) $(\alpha \rightarrow \beta) \equiv \sim(\alpha \wedge \sim \beta)$ (i) $\wedge \phi \rightarrow \phi$

(ii) $\wedge x_{k} \phi \rightarrow \bigvee x_{k} \phi$

(iii) $\wedge x_{k} \phi \equiv \phi$, if $x_{k} \notin F V(\phi)$

(iv) $\vee x_{k} \phi \equiv \phi$, if $x_{k} \notin F V(\phi)$

(v) $\wedge x_{k}(\phi \rightarrow \psi) \equiv\left(\phi \rightarrow \wedge x_{k} \psi\right)$, if $x_{k} \notin F V(\phi)$

(vi) $\vee x_{k}(\phi \rightarrow \psi) \equiv\left(\phi \rightarrow \bigvee x_{k} \psi\right)$, if $x_{k} \notin F V(\phi)$

(vii) $\wedge x_{k}(\phi \rightarrow \psi) \equiv\left(\vee x_{k} \phi \rightarrow \psi\right)$, if $x_{k} \notin F V(\psi)$

(viii) $\vee x_{k} \sim \phi \equiv \sim \wedge x_{k} \phi$

(ix) $\alpha \rightarrow \mathrm{V} x_{k} \alpha$

(x) $\left(\phi \rightarrow \wedge x_{k} \psi\right) \rightarrow\left[\left(\bigwedge x_{k} \psi \rightarrow \phi\right) \rightarrow(\phi \rightarrow \psi)\right]$

(xi) $\left(\mathrm{V} x_{k} \phi \rightarrow \psi\right) \rightarrow\left[\left(\psi \rightarrow \bigvee x_{k} \phi\right) \rightarrow(\phi \rightarrow \psi)\right]$

(xii) $\phi x_{k} / t_{s} \rightarrow \bigvee x_{k} \phi$, if $x_{k} \in F f\left(t_{s}, \phi\right)$

(xiii) $\wedge x_{k}(\phi \rightarrow \psi) \rightarrow\left(\wedge x_{k} \phi \rightarrow \wedge x_{k} \psi\right)$

(xiv) $\wedge x_{k}(\phi \rightarrow \psi) \rightarrow\left(\vee x_{k} \phi \rightarrow \bigvee x_{k} \psi\right)$

(xv) $\wedge x_{k}(\alpha \equiv \beta) \rightarrow\left(\wedge x_{k} \alpha \equiv \wedge x_{k} \beta\right)$

(xvi) $\wedge x_{k}(\alpha \equiv \beta) \rightarrow\left(\vee x_{k} \alpha \equiv \bigvee x_{k} \beta\right)$

(xvii) $\sim \vee x_{k} \sim(\phi \rightarrow \psi) \equiv\left(\bigvee x_{k} \phi \rightarrow \psi\right)$,if

$x_{k} \notin F V(\psi)$

(xviii) $\wedge x_{k}(\phi \wedge \psi) \equiv\left(\wedge x_{k} \phi \wedge \wedge x_{k} \psi\right)$

(xix) $\left(\wedge x_{k} \phi \vee \wedge x_{k} \psi\right) \rightarrow \wedge x_{k}(\phi \vee \psi)$

(xx) $\vee x_{k}(\phi \rightarrow \psi) \equiv\left(\wedge x_{k} \phi \rightarrow \vee x_{k} \psi\right)$

(xxi) $\vee x_{k}(\phi \wedge \psi) \rightarrow\left(\bigvee x_{k} \phi \wedge \vee x_{k} \psi\right)$

(xxii) $\vee x_{k}(\phi \vee \psi) \equiv\left(\vee x_{k} \phi \vee \vee x_{k} \psi\right)$

(xxiii) $\wedge x_{k}(\phi \vee \psi) \equiv\left(\phi \vee \wedge x_{k} \psi\right)$, if

$x_{k} \notin F V(\phi)$

(xxiv) $\wedge x_{k}(\phi \rightarrow \psi) \equiv\left(\vee x_{k} \phi \rightarrow \psi\right)$, if

$x_{k} \notin F V(\psi)$

$(\mathrm{xxv}) \vee x_{k}(\phi \wedge \psi) \equiv\left(\phi \wedge \vee x_{k} \psi\right)$, if $x_{k} \notin F V(\phi)$

(xxvi) $\wedge x_{k} \wedge x_{s} \phi \equiv \wedge x_{s} \wedge x_{k} \phi$

(xxvii) $\vee x_{k} \vee x_{s} \phi \equiv \vee x_{s} \vee x_{k} \phi$

(xxviii) $\vee x_{k} \wedge x_{s} \phi \rightarrow \wedge x_{s} \vee x_{k} \phi$

(xxix) $\sim \vee x_{k} \phi \equiv \wedge x_{k} \sim \phi$

$(\mathrm{xxx}) \sim \vee x_{k} \sim \phi \equiv \wedge x_{k} \phi$

(xxxi) $\sim \wedge x_{k} \sim \phi \equiv \bigvee x_{k} \phi$

(xxxii) $\left\{\left[\left(\psi^{*} \rightarrow \phi^{*}\right) \rightarrow \phi^{*}\right] \rightarrow \phi^{*}\right\} \rightarrow(\wedge \phi \rightarrow \psi)$

(xxxiii) $\sim Q_{i}(\wedge \phi \wedge \psi) \equiv\left(\wedge \phi \rightarrow \sim Q_{i} \psi\right)$

(xxxiv) $\wedge x_{k}(\phi \equiv \psi) \rightarrow\left[\wedge x_{k}(\phi \rightarrow \psi) \wedge\right.$

$\left.\wedge x_{k}(\psi \rightarrow \phi)\right]$.

Using Definition 8.1, Corollary 8.3, Corollary 8.4 and using the proof of Theorem 4.2 (see [20], pp.222 - 
224), one can obtain

Corollary 8.5 (on the extensionality of logical expressions). Let $x_{1}, \ldots, x_{n}, y_{1}, \ldots, y_{l}$ be all the free variables, which occur in the expressions $\alpha$ and $\beta$, and let $C^{\alpha}$ be any expression that contains $\alpha$ or an expression obtained from $\alpha$ by the substitution for the variables $x_{1}, \ldots, x_{n}$ of some other variables different from the bound variables occurring in the expressions $\alpha$ or $\beta$, and let $C^{\beta}$ differ from $C^{\alpha}$ only in that in certain places (unnecessarily in all these places) in which in $C^{\alpha}$ there occurs $\alpha$ or an expression obtained from $\alpha$ by a substitution for the variables $x_{1}, \ldots, x_{n}$, in the corresponding places in $C^{\beta}$ there occurs $\beta$ or an expression obtained from $\beta$ by an appropriate substitution, while the variables $y_{1}, \ldots, y_{l}$ are all the free variables in $C^{\alpha}$ and $C^{\beta}$. Then the sentence:

$$
\wedge \ldots y_{1}, \ldots, y_{l}\left(\wedge x_{1}, \ldots, x_{n}(\alpha \equiv \beta) \rightarrow\left(C^{\alpha} \equiv C^{\beta}\right)\right)
$$

is a theorem in $L_{D}$.

Definition 8.6. Let $\phi \in S_{1}$ and $\alpha \in S m p$. Next let $v: A t_{0} \rightarrow\left|\mathfrak{M}_{2}\right|$ be an arbitrary, but fixed valuation in the matrix $\mathfrak{M}_{2}$ such that $h^{v}(j(\phi))=1$. Then

$$
e_{\phi}(\alpha)=\left\{\begin{array}{lll}
\wedge \phi \wedge \alpha, & \text { if } & v(\mathfrak{i}(\alpha))=0 \\
\wedge \phi \rightarrow \alpha, & \text { if } & v(\mathfrak{i}(\alpha))=1
\end{array}\right.
$$

By the definition of the formulas $\phi^{*}, \psi^{*}$, one can easily obtain right away

\section{Corollary 8.7.}

$\left(\forall \phi, \psi \in S_{1}\right)\left(\exists e \in \varepsilon_{*}^{1}\right)\left[h^{e}(\Lambda \phi) \in L_{D} \mathbb{V}\right.$

$\left.h^{e}\left(\left(\psi^{*} \rightarrow \phi^{*}\right) \rightarrow \phi^{*}\right) \in L_{D}\right]$.

Lemma 8.8. If $\wedge \phi \rightarrow \psi \in L_{D}$, then

$$
\begin{aligned}
& \quad\left(\forall e \in \varepsilon_{*}^{1}\right)\left[h^{e}(\Lambda \phi) \in L_{D} \Rightarrow h^{e}(\psi) \in L_{D} \&\right. \\
& \left.P_{1}\left(h^{e}(\Lambda \phi)\right) \subseteq P_{1}\left(h^{e}(\psi)\right)\right] \text { and } \\
& \left(\forall e \in \varepsilon_{*}^{1}\right)\left[h^{e}\left(\left(\psi^{*} \rightarrow \phi^{*}\right) \rightarrow \phi^{*}\right) \in L_{D} \Rightarrow\right. \\
& \left.h^{e}\left(\phi^{*}\right) \in L_{D} \& P_{1}\left(h^{e}\left(\psi^{*}\right)\right) \subseteq P_{1}\left(h^{e}\left(\phi^{*}\right)\right)\right] .
\end{aligned}
$$

Proof. Let (1) $\wedge \phi \rightarrow \psi \in L_{D}$. Hence, by Corollary 8.3, we obtain that (2) $\left(\forall e \in \varepsilon_{*}^{1}\right)\left[h^{e}(\Lambda \phi) \in L_{D} \Rightarrow\right.$ $\left.h^{e}(\psi) \in L_{D}\right]$. Hence, by the definition of the set $L_{D}$ and by the definition of the matrix $\mathfrak{M}_{D}$, it follows that (3) $\left(\forall e \in \varepsilon_{*}^{1}\right)\left[h^{e}(\Lambda \phi) \in L_{D} \Rightarrow h^{e}(\psi) \in L_{D}\right.$ \&

$$
\left.P_{1}\left(h^{e}(\Lambda \phi)\right) \subseteq P_{1}\left(h^{e}(\psi)\right)\right] \text {. Let (4) } F V(\phi)=
$$

$\left\{x_{1}, \ldots, x_{n}\right\}$ and (5) $F V(\psi)=\left\{y_{1}, \ldots, y_{m}\right\}$. Hence, by the definition of the formulas $\phi^{*}, \psi^{*}$, it follows that (6) $\phi^{*}=\bigvee x_{1} \ldots \vee x_{n} \sim \phi$ and

(7) $\psi^{*}=\vee y_{1} \ldots \vee y_{m} \sim \psi$.

Hence, from (1), by Definition 8.1, Corollary 8.3, Corollary 8.4 and Corollary 8.5, we obtain that (8) $\psi^{*} \rightarrow \phi^{*} \in L_{D}$. Hence, by Definition 8.1, Corollary 8.3, Corollary 8.4 and Corollary 8.5, we obtain that (9) $\left(\forall e \in \varepsilon_{*}^{1}\right)\left[h^{e}\left(\left(\psi^{*} \rightarrow \phi^{*}\right) \rightarrow \phi^{*}\right) \in L_{D} \Rightarrow\right.$ $\left.h^{e}\left(\phi^{*}\right) \in L_{D} \quad \& P_{1}\left(h^{e}\left(\psi^{*}\right)\right) \subseteq P_{1}\left(h^{e}\left(\phi^{*}\right)\right)\right]$, what together with (3) complete the proof.

Lemma 8.9. If $\phi \in S_{1}^{*}$ and $\left(\exists e \in \varepsilon_{*}^{1}\right)\left[h^{e}(\phi) \in\right.$ $\left.L_{D}\right]$, then $h^{e} \phi(\phi) \in L_{D}$.

Proof. Now we assume that (1) $Q_{i} \in\left\{\bigwedge x_{i}, \bigvee x_{i}\right\}$ and (2) $\phi \in S_{1}^{*}$ and (3) $\left(\exists e_{1} \in \varepsilon_{*}^{1}\right)\left[h^{e_{1}}(\phi) \in L_{D}\right]$. Hence, by the definition of the set $L_{D}$, it follows that (4) $\left(\exists v: A t_{0} \rightarrow\left|\mathfrak{M}_{2}\right|\right)\left[h^{v}(j(\phi))=1\right]$.

Let:

(1.1) $\phi \in S m p$.

Hence, by (4) and Definition 8.6, one can obtain that

$$
h^{e_{\phi}}(\phi)=\wedge \phi \rightarrow \phi .
$$

Hence, by Corollary 8.4 (i), in (1.1), it follows that

$$
h^{e_{\phi}}(\phi) \in L_{D} \text {. }
$$

Let

(1.2) $\phi=\sim P_{k}^{n}\left(t_{1}, \ldots, t_{n}\right)$.

Hence, from (4) and Definition 8.6, it follows that

$$
h^{e_{\phi}}(\phi)=\sim\left(\wedge \phi \wedge P_{k}^{n}\left(t_{1}, \ldots, t_{n}\right)\right) .
$$

Therefore, by Corollary 8.4 (107) and (1.2), it follows that

$$
h^{e} \phi(\phi) \equiv(\Lambda \phi \rightarrow \phi) \in L_{D} .
$$

So, using Corollary 8.4 (i), in (1.2), one can obtain that

$$
h^{e_{\phi}}(\phi) \in L_{D}
$$

Let

$$
\phi=\phi_{1} \vee \phi_{2}
$$

and assume inductively that

$$
\left(a_{1}\right) h^{e_{\phi}}\left(\phi_{1}\right) \in L_{D}
$$

or

$$
\left(a_{2}\right) h^{e_{\phi}}\left(\phi_{2}\right) \in L_{D} .
$$


From Definition 8.6 it follows that

$$
h^{e_{\phi}}\left(\phi_{1} \vee \phi_{2}\right)=h^{e_{\phi}}\left(\phi_{1}\right) \vee h^{e_{\phi}}\left(\phi_{2}\right) \text {. }
$$

Next, in $\left(a_{1}\right)$ and $\left(a_{2}\right)$, from (1.3) and by Definition 8.6, it follows that

$$
P_{1}\left(h^{e_{\phi}}\left(\phi_{1}\right)\right)=P_{1}\left(h^{e} \phi\left(\phi_{2}\right)\right)=P_{1}(\phi) .
$$

Hence, from (10), by Corollary 8.4 (40) and Corollary $8.4(41)$, in $\left(a_{1}\right)$ and $\left(a_{2}\right)$, in (1.3), it follows that

$$
h^{e_{\phi}}(\phi) \in L_{D}
$$

Let

(1.4) $\phi=\phi_{1} \wedge \phi_{2}$

and assume inductively that

$$
h^{e_{\phi}}\left(\phi_{1}\right), h^{e_{\phi}}\left(\phi_{2}\right) \in L_{D} \text {. }
$$

From (1.4) and (13), using Definition 8.6, by Corollary 8.4 (62), in (1.4), one can obtain that

$$
h^{e_{\phi}}(\phi) \in L_{D} \text {. }
$$

Let

$$
\phi=Q_{i} \phi^{\prime}
$$

and assume inductively that

$$
h^{e_{\phi}}\left(\phi^{\prime}\right) \in L_{D}
$$

Hence, from (1.5), using Definition 8.6, by Corollary 8.4 (ix) and Corollary 8.3, in (1.5), one can obtain that

$$
h^{e} \phi(\phi) \in L_{D},
$$

which completes the proof. $\square$

Lemma 8.10. If $\phi \in S_{1}$ and

$\left(\exists e \in \varepsilon_{*}^{1}\right)\left[h^{e}(\phi) \in L_{D}\right]$, then $h^{e_{\phi}}(\phi) \in L_{D}$.

Proof. By Definition 8.6, Corollary 8.4, Corollary 8.5

and Lemma 8.9 and by the well-known Theorem concerning normal form (see [19] pp. 35-42 and 130-132, [20] pp. 214 - 222, and [37] pp.146-149).

Lemma 8.11. Let $\wedge \phi \rightarrow \psi \in L_{2}$ and

$$
\begin{gathered}
\left(\forall e \in \varepsilon_{*}^{1}\right)\left[h^{e}(\Lambda \phi) \in L_{D} \Rightarrow h^{e}(\psi) \in L_{D} \&\right. \\
\left.P_{1}\left(h^{e}(\phi)\right) \subseteq P_{1}\left(h^{e}(\psi)\right)\right] \text { and } \\
\left(\forall e \in \varepsilon_{*}^{1}\right)\left[h^{e}\left(\left(\psi^{*} \rightarrow \phi^{*}\right) \rightarrow \phi^{*}\right) \in L_{D} \Rightarrow\right. \\
\left.h^{e}\left(\phi^{*}\right) \in L_{D} \& P_{1}\left(h^{e}\left(\psi^{*}\right)\right) \subseteq P_{1}\left(h^{e}\left(\phi^{*}\right)\right)\right] . \text { Then } \\
\wedge \phi \rightarrow \psi \in L_{D} .
\end{gathered}
$$

Proof. By Theorem 7.2, the Definition 8.1, Corollary 8.3, Corollary 8.4, Corollary 8.5, Definition 8.6, Corollary 8.7 , Lemma 8.10 and by the definition of the matrix $\mathfrak{M}_{D}$ and by the definitions of the formulas $\phi^{*}, \psi^{*}$

Lemma 8.12. Let $\phi, \psi \in S_{1}$ and

$$
\left(\exists e \in \varepsilon_{*}^{1}\right)\left[h^{e}(\phi) \in L_{D}\right]
$$

and

$$
\begin{gathered}
\left(\forall e \in \varepsilon_{*}^{1}\right)\left[h^{e}(\phi) \in L_{D} \Rightarrow h^{e}(\psi) \in L_{D} \&\right. \\
\left.P_{1}\left(h^{e}(\phi)\right) \subseteq P_{1}\left(h^{e}(\psi)\right)\right] .
\end{gathered}
$$

Then

$\left(\forall e \in \varepsilon_{*}^{1}\right)\left[h^{e}(\phi) \in L_{2} \Rightarrow h^{e}(\psi) \in L_{2}\right]$.

Proof. Let (1) $\phi, \psi \in S_{1}$, (2) $\left(\exists e \in \varepsilon_{*}^{1}\right)\left[h^{e}(\phi) \in L_{D}\right]$ and (3) $\left(\forall e \in \varepsilon_{*}^{1}\right)\left[h^{e}(\phi) \in L_{D} \Rightarrow\right.$

$$
\left.h^{e}(\psi) \in L_{D} \& P_{1}\left(h^{e}(\phi)\right) \subseteq P_{1}\left(h^{e}(\psi)\right)\right] .
$$

From (1), (2), it follows that

$\left(\exists e_{1} \in \varepsilon_{*}^{1}\right)\left[h^{e_{1}}(\phi) \in L_{2}\right]$. Now suppose that (5) $\left(\exists e_{2} \in \varepsilon_{*}^{1}\right)\left[h^{e_{2}}(\phi) \in L_{2} \& h^{e_{2}}(\psi) \notin L_{2}\right]$. Next assume that $(6) h^{e_{2}}(\phi)=\phi^{\prime}$ and (7) $h^{e_{2}}(\psi)=\psi^{\prime}$. From (5) - (7), it follows that (8) $\phi^{\prime} \in L_{2}$ and (9) $\psi^{\prime} \notin L_{2}$. From (8) it follows that (10) $\left(\exists e \in \varepsilon_{*}^{1}\right)\left[h^{e}\left(\phi^{\prime}\right) \in L_{D}\right]$. Hence, by Lemma 8.10, it follows that (11) $h^{e} \phi^{\prime}\left(\phi^{\prime}\right) \in L_{D}$. Hence, from (3) it follows that (12) $h^{e} \phi^{\prime}\left(\psi^{\prime}\right) \in L_{D}$. From (8) and (9) and Definition 8.6 and Theorem 4.2, it follows that (14) $h^{e} \phi^{\prime}\left(\psi^{\prime}\right) \notin L_{2}$. From (12), by the definition of the set $L_{D}$, it follows that $(15) h^{e} \phi^{\prime}\left(\psi^{\prime}\right) \in L_{2}$, what contradicts (14).

Lemma 8.13. Let

$$
\begin{gathered}
\left(\forall e \in \varepsilon_{*}^{1}\right)\left[h^{e}\left(\left(\psi^{*} \rightarrow \phi^{*}\right) \rightarrow \phi^{*}\right) \in L_{D} \Rightarrow\right. \\
\left.h^{e}\left(\phi^{*}\right) \in L_{D} \& P_{1}\left(h^{e}\left(\psi^{*}\right)\right) \subseteq P_{1}\left(h^{e}\left(\phi^{*}\right)\right)\right]
\end{gathered}
$$

and

$\left(\exists e \in \varepsilon_{*}^{1}\right)\left[h^{e}\left(\left(\psi^{*} \rightarrow \phi^{*}\right) \rightarrow \phi^{*}\right) \in L_{D}\right]$.

Then

$$
\begin{gathered}
\left(\forall e \in \varepsilon_{*}^{1}\right)\left[h^{e}\left(\left(\psi^{*} \rightarrow \phi^{*}\right) \rightarrow \phi^{*}\right) \in L_{2} \Rightarrow\right. \\
\left.h^{e}\left(\phi^{*}\right) \in L_{2}\right] .
\end{gathered}
$$

Proof. The proof of this lemma is analogical to the proof of Lemma 8.12.

In [50] we have proved the following Lemma:

Lemma 8.14. Let $\phi, \psi \in S_{1}, X \subseteq S_{1}$ and

$$
\begin{aligned}
\left(\exists v: A t_{0} \rightarrow\right. & \left.\left|\mathfrak{M}_{2}\right|\right)\left[h^{v}(j(\phi))=1\right] \text { and } \\
& \operatorname{Cn}_{1}\left(R_{0+}, L_{2} \cup X\right)=Z_{3}
\end{aligned}
$$

and

$\left(\forall e \in \varepsilon_{*}^{1}\right)\left[h^{e}(\phi) \in Z_{3} \Rightarrow h^{e}(\psi) \in Z_{3}\right]$. 
Then $\wedge \phi \rightarrow \psi \in Z_{3}$.

In consequence:

Lemma 8.15. If $\left(\exists e \in \varepsilon_{*}^{1}\right)\left[h^{e}(\phi) \in L_{D}\right]$ and $\left(\forall e \in \varepsilon_{*}^{1}\right)\left[h^{e}(\phi) \in L_{D} \Rightarrow h^{e}(\psi) \in L_{D} \&\right.$ $\left.P_{1}\left(h^{e}(\phi)\right) \subseteq P_{1}\left(h^{e}(\psi)\right)\right]$, then $\Lambda \phi \rightarrow \psi \in L_{2}$.

Proof. By Corollary 8.4, Lemma 8.12 and Lemma 8.14.

\section{Lemma 8.16. Let}

$$
\begin{gathered}
\left(\forall e \in \varepsilon_{*}^{1}\right)\left[h^{e}\left(\left(\psi^{*} \rightarrow \phi^{*}\right) \rightarrow \phi^{*}\right) \in L_{D} \Rightarrow\right. \\
\left.h^{e}\left(\phi^{*}\right) \in L_{D} \& P_{1}\left(h^{e}\left(\psi^{*}\right)\right) \subseteq P_{1}\left(h^{e}\left(\phi^{*}\right)\right)\right]
\end{gathered}
$$

and

$\left(\exists e \in \varepsilon_{*}^{1}\right)\left[h^{e}\left(\left(\psi^{*} \rightarrow \phi^{*}\right) \rightarrow \phi^{*}\right) \in L_{D}\right]$.

Then $\psi^{*} \rightarrow \phi^{*} \in L_{2}$.

Proof. By Corollary 8.4, Lemma 8.13 and Lemma 8.14 .

Lemma 8.17. Let $\phi^{*}, \psi^{*} \in S_{1}$.

If $\left(\forall e \in \varepsilon_{*}^{1}\right)\left[h^{e}\left(\left(\psi^{*} \rightarrow \phi^{*}\right) \rightarrow \phi^{*}\right) \in L_{D} \Rightarrow\right.$ $\left.h^{e}\left(\phi^{*}\right) \in L_{D} \& P_{1}\left(h^{e}\left(\psi^{*}\right)\right) \subseteq P_{1}\left(h^{e}\left(\phi^{*}\right)\right)\right]$ and

$\left(\forall e \in \varepsilon_{*}^{1}\right)\left[h^{e}(\phi) \in L_{D} \Rightarrow h^{e}(\psi) \in L_{D} \&\right.$ $\left.P_{1}\left(h^{e}(\phi)\right) \subseteq P_{1}\left(h^{e}(\psi)\right)\right]$, then $\wedge \phi \rightarrow \psi \in L_{D}$.

Proof. By the definitions of the formulas $\phi^{*}, \psi^{*}$ andCorollary 8.7, Lemma 8.11, Lemma 8.15 and Lemma 8.16.

Lemma 8.18. Let $\phi^{*}, \psi^{*} \in S_{0}$.

$$
\begin{aligned}
& I f\left(\forall e \in \varepsilon_{*}^{0}\right)\left[h^{e}\left(\left(\psi^{*} \rightarrow \phi^{*}\right) \rightarrow \phi^{*}\right) \in T_{D} \Rightarrow\right. \\
& \left.h^{e}\left(\phi^{*}\right) \in T_{D} \& P_{0}\left(h^{e}\left(\psi^{*}\right)\right) \subseteq P_{0}\left(h^{e}\left(\phi^{*}\right)\right)\right] \text { and } \\
& \quad\left(\forall e \in \varepsilon_{*}^{0}\right)\left[h^{e}(\phi) \in T_{D} \Rightarrow h^{e}(\psi) \in T_{D} \&\right. \\
& \left.P_{0}\left(h^{e}(\phi)\right) \subseteq P_{0}\left(h^{e}(\psi)\right)\right] \text {, then } \phi \rightarrow \psi \in T_{D} .
\end{aligned}
$$

Proof. Using the similar reasoning as in the proof of Lemma 8.17.

Lemma 8.19.If $\phi \rightarrow \psi \in T_{D}$, then

$$
\begin{gathered}
\left(\forall e \in \varepsilon_{*}^{0}\right)\left[h^{e}(\phi) \in T_{D} \Rightarrow h^{e}(\psi) \in T_{D} \&\right. \\
\left.P_{0}\left(h^{e}(\phi)\right) \subseteq P_{0}\left(h^{e}(\psi)\right)\right]
\end{gathered}
$$

and

$$
\begin{gathered}
\left(\forall e \in \varepsilon_{*}^{0}\right)\left[h^{e}\left(\left(\psi^{*} \rightarrow \phi^{*}\right) \rightarrow \phi^{*}\right) \in T_{D} \Rightarrow\right. \\
\left.h^{e}\left(\phi^{*}\right) \in T_{D} \& P_{0}\left(h^{e}\left(\psi^{*}\right)\right) \subseteq P_{0}\left(h^{e}\left(\phi^{*}\right)\right)\right] .
\end{gathered}
$$

Proof. The proof of this Lemma is analogical to the proof of Lemma 8.8.

\section{The Main Result}

Theorem 9.1. $\left\langle R_{0}, T_{D}\right\rangle \in$ Syst $\cap A_{0}$.
Proof. By Lemma 8.18 and Lemma 8.19. $\square$

Theorem 9.2. $\left\langle R_{0+}, L_{D}\right\rangle \in$ Syst $\cap A_{1}$.

Proof. By Lemma 8.8 and Lemma 8.17.

Theorem 9.3. $\left\langle R_{0+}, L_{2}\right\rangle \in$ Syst $\cap C_{1}$.

Proof. By similar reasonings as in the proofs of

Lemma 8.8 and Lemma 8.17 (or by Corollary 8.7, the definition of the set $L_{D}$ and by Lemma 8.14).

Theorem 9.4. $\left\langle R_{0}, T_{D}\right\rangle \in$ Syst $\cap A I N C$.

Proof. By the definition of the set $T_{D}$ and by the Definition 6.1 and the Definition 6.2.

Theorem 9.5. $\left\langle R_{0+}, L_{D}\right\rangle \in$ Syst $\cap$ AINC.

\section{Proof. Let}

(1) $\alpha \in S_{1}$

and

(2) $\beta \in S_{1}$.

Hence, by the definition of the $\operatorname{set} L_{D}$, it follows that

(3) $\alpha \rightarrow(\sim \alpha \rightarrow \beta) \in L_{D}$, where

(4) $P_{1}(\beta) \subseteq P_{1}(\alpha)$.

From (1)-(4), it follows that

(5) $S_{1 \alpha} \subseteq C n\left(R_{0+}, L_{D} \cup\{\alpha, \sim \alpha\}\right)$.

Let now,

(6) $\quad P_{1}(\beta) \nsubseteq P_{1}(\alpha)$.

Next, by the definition of the set $L_{2}$, it follows that

(7) $(\alpha \wedge \sim \alpha) \rightarrow(\beta \wedge \sim \beta) \in L_{2}$.

Next, from (6), by the definition of the set $T_{D}$, it follows that

(8) $j(\alpha \wedge \sim \alpha) \rightarrow j(\beta \wedge \sim \beta) \notin T_{D}$.

Hence, from (6), (7), by the definition of the set $L_{D}$, it follows that

(9) $\beta \notin C n\left(R_{0+}, L_{D} \cup\{\alpha \wedge \sim \alpha\}\right)$

or

(10) $\sim \beta \notin \operatorname{Cn}\left(R_{0+}, L_{D} \cup\{\alpha \wedge \sim \alpha\}\right)$,

what together with 5), 6) and the Definition 6.2, completes the proof.

\section{Summary}

Remark 10.1. Let $\left(\forall e \in \varepsilon_{*}^{0}\right)\left[h^{e}\left(\psi^{*}\right) \in L \Rightarrow\right.$ $\left.h^{e}\left(\phi^{*}\right) \in L \& P_{0}\left(h^{e}\left(\psi^{*}\right)\right) \subseteq P_{0}\left(h^{e}\left(\phi^{*}\right)\right)\right]=\Lambda_{0}$ and

$$
\left(\forall e \in \varepsilon_{*}^{0}\right)\left[h^{e}\left(\left(\psi^{*} \rightarrow \phi^{*}\right) \rightarrow \phi^{*}\right) \in L \Rightarrow\right.
$$


$\left.h^{e}\left(\phi^{*}\right) \in L \& P_{0}\left(h^{e}\left(\psi^{*}\right)\right) \subseteq P_{0}\left(h^{e}\left(\phi^{*}\right)\right)\right]=\Lambda_{1}$.

By an inspection of Definition 5.1, Definition 5.2, Definition 7.1, Lemma 8.18 and Lemma 8.19, one can easily see that in condition (2) of Definition 5.1 one cannot put $\Lambda_{0}$ instead of $\Lambda_{1}$.

\section{Remark 10.2.}

Let $\left(\forall e \in \varepsilon_{*}^{1}\right)\left[h^{e}\left(\psi^{*}\right) \in L \Rightarrow h^{e}\left(\phi^{*}\right) \in\right.$

$\left.L \& P_{1}\left(h^{e}\left(\psi^{*}\right)\right) \subseteq P_{1}\left(h^{e}\left(\phi^{*}\right)\right)\right]=\Lambda_{0}$

and

$$
\begin{gathered}
\left(\forall e \in \varepsilon_{*}^{1}\right)\left[h^{e}\left(\left(\psi^{*} \rightarrow \phi^{*}\right) \rightarrow \phi^{*}\right) \in L \Rightarrow\right. \\
\left.h^{e}\left(\phi^{*}\right) \in L \& P_{1}\left(h^{e}\left(\psi^{*}\right)\right) \subseteq P_{1}\left(h^{e}\left(\phi^{*}\right)\right)\right]=\Lambda_{1} .
\end{gathered}
$$

By an inspection of Definition 5.3 and Definition 5.4 and Definition 8.1 and Lemma 8.8 and Lemma 8.17 , one can easily see that in condition (2) of Definition 5.3 one cannot put $\Lambda_{0}$ instead of $\Lambda_{1}$.

\section{Remark 10.3.}

$$
\begin{gathered}
\operatorname{Let}\left(\forall e \in \varepsilon_{*}^{1}\right)\left[h^{e}\left(\psi^{*}\right) \in L \Rightarrow h^{e}\left(\phi^{*}\right) \in L\right]=\Lambda_{0} \\
\text { and }\left(\forall e \in \varepsilon_{*}^{1}\right)\left[h^{e}\left(\left(\psi^{*} \rightarrow \phi^{*}\right) \rightarrow \phi^{*}\right) \in L \Rightarrow\right. \\
\left.h^{e}\left(\phi^{*}\right) \in L\right]=\Lambda_{1} .
\end{gathered}
$$

By an inspection of Definition 3.1, Definition 3.2 and by Lemma 8.14 , Theorem 9.3, one can easily see that in condition (2) of Definition 3.1 one cannot put $\Lambda_{0}$ instead of $\Lambda_{1}$.

Using Definition 2.2 and Definition 6.1 and Definition 6.2, one can obtain the following remark:

\section{Remark 10.4.}

$$
\langle R, X\rangle \in \text { Syst } \cap A I N C \Rightarrow\langle R, X\rangle \in \mathrm{Cns}^{A},
$$
where $R \subseteq R_{S_{i}}$ and $X \subseteq S_{i}$ and $i \in\{0,1\}$.

\section{References}

[1] W. Ackerman. Begrundung einer strengen implication. The Journal of Symbolic Logic, 21:113-128, 1956.

[2] A. R. Anderson. Completeness theorems for the system E of entailment and EQ of entailment with quantification. Zeitschrift für mathematische Logik und Grundlagen der Mathematik, 6:201-216, 1960.

[3] A. R. Anderson and N. D. Belnap. The Pure Calculus of Entailment. The Journal of Symbolic Logic, 27:19-52,
1962.

[4] A. R. Anderson and N. D. Belnap. First degree entailments. Mathematische Annalen, 149:302-319, 1963.

[5] A. R. Anderson and N. D. Belnap. Entailment: The Logic of Relevance and Necessity, volume I. Princeton University Press, 1975.

[6] A. R. Anderson, N. D. Belnap, and J. M. Dunn. Entailment: The Logic of Relevance and Necessity, volume II. Princeton University Press, 1992.

[7] R. Barcan-Marcus. Strict implication, Deducibility and the deduction theorem. The Journal of Symbolic Logic, 18:234-236, 1953.

[8] J. Beall. Truth without Detachment, 2012. A Talk delivered at Munich Center for Mathematical Philosophy, 14.03.2012.

[9] K. Bimbo. Relevance Logics and Relation Algebras. In Philosophy of Logic, ed. D. Jacquette, vol. 5, pages 723-789. Elsevier/North-Holland, Amsterdam, 2007. Included in series Handbook of the Philosophy of Science (eds. D. M.Gabbay, P. Thagard and J. Woods).

[10] K. Bimbo, J. M. Dunn, and R. D. Maddux. Relevance Logics and Relation Algebras. The Review of Symbolic Logic, 2:102-131, 2009.

[11] L. Borkowski.Comments on Conditional Sentences and on Material and Strict Implication, inThe Book in Honour of Kazimierz Ajdukiewicz., 102-104, Warszawa, 1964.

[12] R. T. Brady. Entailment, classicality and the paradoxes, delivered to the Australasian Association of Philosophy Conference, A.N.U., Canberra, 1989.

[13] Ross T. Brady. Relevant implication and the case for a weaker logic. Journal of Philosophical Logic, 25:151-183, 1996.

[14] Jingde Cheng. The fundamental role of entailment in knowledge representation and reasoning. Journal of Computing and Information, 2:853-873, 1996.

[15] Jingde Cheng. Temporal relevant logic as the logic basis for reasoning about dynamics of concurrent systems. In Proceedings of the 1998 IEEE-SMC Annual International Conference on Systems, Man, and Cybernetics, San Diego, USA, 11-14 October 1998, 1998

[16] Z. Czerwinski. On paradox of implication. Studia Logica, VII:264-271, 1958.

[17] A. E. Duncan-Jones. Is strict implication the same as entailment? Analysis, II:70-78, 1935.

[18] J. M. Dunn. A modification of Parry's analytic 
implication. Notre Dame Journal of Formal Logic, 13:195-205, 1972.

[19] Yu. L. Ershov and E. A. Palyutin. Mathematical Logic. Mir Publishers, MOSCOW, 1984.

[20] A. Grzegorczyk. An Outline of Mathematical Logic. Fundamental Results and Notions Explained with All Details, D. Reidel Publishing Company, Dordrecht-Holland/Boston-USA, PWN, Warszawa, 1974.

[21] I. Hacking. what is strict implication ? The Journal of Symbolic Logic, 28:51-71, 1963.

[22] R. Hirsch and Szabolc Mikulas. Positive Fragments of Relevance Logic and Algebras of Binary Relations. The Review of Symbolic Logic, 4:81-105, 2011.

[23] Anthony Hunter and S. Konieczny. Approaches to measuring inconsistent information. In Inconsistency Tolerance, 191-236, Springer, 2005.

[24] S. A. Kripke. The problem of entailment. The Journal of Symbolic Logic, 24:324, 1959.

[25] C. I. Lewis. Implication and the algebra of logic. Mind, New Series, 21:522-531, 1912.

[26] C. Lewy. Entailment. Aristotelian Society Supplementary Volume, 32:123-142, 1958.

[27] S. Mccall. A new variety of implication. The Journal of Symbolic Logic, 29:151-152, 1964.

[28] R. K. Meyer. Entailment and relevant implication. Logique et Analyse, 11:472-479, 1968.

[29] R. K. Meyer. Two questions from Anderson and Belnap. Reports on Mathematical Logic, 7:71-86, 1976.

[30] R. K. Meyer and J. M. Dunn. Entailment and material implication. Notices of The American Mathematical Society, 15:1021-1022, 1966.

[31] R. K. Meyer and R. Routley. Classical relevant logics (I). Studia Logica, XXXII:51-68, 1973.

[32] J. M. MÉndez and G. Robles. Relevance Logics, Paradoxes of Consistency and the K Rule II. A Non-constructive Negation. Logics and Logical Philosophy, 15:175-191, 2006.

[33] J. M. MÉndez, Francisco Salto, and G. Robles. Anderson and Belnap's Minimal Positive Logic with Minimal Negation. Reports on Mathematical Logic, 36:117-130, 2002.

[34] J. Norman and R. Sylvan, Editors. Directions in Relevant Logic. Kluwer Academic Publishers, 1989.

[35] R. Z. Parks and J. R. Chidgey. Necessity and ticket entailment. Notre Dame Journal of Formal Logic,
13:224-226, 1972.

[36] W. A. Pogorzelski. The Classical Propositional Calculus. PWN, Warszawa, 1975.

[37] W. A. Pogorzelski. The Classical Calculus of Quantifiers. PWN, Warszawa, 1981.

[38] W. A. Pogorzelski and T. Prucnal. The substitution rule for predicate letters in the first-order predicate calculus. Reports on Mathematical Logic, 5:77-90,1975.

[39] G. Pottinger. On analysing relevance constructively. Studia Logica, 38:171-185, 1979.

[40] G. Priest. Sense, Entailment and Modus Ponens. Journal of Philosophical Logic, 9:415-435, 1980.

[41] A. N. Prior. Facts, propositions, and entailment. Mind, 57:62-68, 1947.

[42] R. Routley. The American Plan Completed: alternative classic-style semantics, without stars, for relevant and paraconsistent logics. Studia Logica, XLIII1/2:131-158, 1984.

[43] R. Routley and R. K. Meyer. Towards a general semantical theory of implication and conditionals. I. Systems with normal conjunctions and disjunctions and aberrant and normal negations. Reports on Mathematical Logic, 4:67-90,1975.

[44] R. Routley and R. K. Meyer. Towards a general semantical theory of implication and conditionals. II. Systems with normal conjunctions and disjunctions and aberrant and normal negations. Reports on Mathematical Logic, 9:47-62,1977.

[45] T. J. Smiley. Entailment and deducibility. Proceedings of The Aristotelian Society, 59:233-254, 1959.

[46] B. Sobocinski. Axiomatization of partial system of three-valued calculus of propositions. The Journal of Computing System, 1:22-55, 1952.

[47] T. Stepien. System $\bar{S}$. Reports on Mathematical Logic, 15:59-65, 1983.

[48] T. Stepien. System $\bar{S}$. Zentralblatt für Mathematik, 471, 1983.

[49] T. Stepien. Logic based on atomic entailment. Bulletin of the Section of Logic, 14:65-71, 1985.

[50] T. Stepien. Derivability. Reports on Mathematical Logic, 33:79-99, 1999.

[51] T. Stepien. Logic Based On Atomic Entailment And Paraconsistency. $11^{\text {th }}$ international Congress Of Logic,Methodology and Philosophy Of Science (August 1999, Krakow, Poland). 
[52] T. J. Stepien and L. T. Stepien. Atomic Entailment and Classical Entailment, The Bulletin of Symbolic Logic, 17:317-318, 2011.

[53] N. Tennant. Perfect validity, entailment and paraconsistency. Studia Logica, XLIII:181-200, 1984.

[54] A. Urquhart. Semantics for relevant logics. The Journal of Symbolic Logic, 37:159-169, 1972.

[55] A. Urquhart. The Undecidability of Entailment and Relevant Implication, The Journal of Symbolic Logic, 49:1059-1073, 1984

[56] A. Urquhart. Failure of Interpolation in Relevant Logics. Journal of Philosophical Logic, 22:449-479, 1993.

[57] A. Urquhart. Anderson and Belnap's Invitation to Sin.
Journal of Philosophical Logic, 39:453-472, 2010.

[58] Andreas Weiermann and Martin Bunder. Ackermannian lower bounds for a combinatorial problem related to the decidability of certain relevant logics, 2005. http://cage.ugent.be/ weierman/relevancelogic.pdf.

[59] P. Weingartner. Two simple ideas of relevance. In Proceedings of the Xxxth Conference on the History of Logic, Dedicated To Roman Suszko, Cracow, 19-21 October 1984, 1984.

[60] G. Xiao and Y. Ma, Inconsistency measurement based on variables in minimal unsatisfable subsets. 2012. A Talk delivered at European Conference on Artificial Intelligence, ECAI'12, France (2012). 